\title{
Comparison of postoperative morbidity between piezoelectric surgery and conventional rotary instruments in mandibular third molar surgery: a split-mouth clinical study
}

\author{
Yakup Gulnahar ${ }^{1}$, Aysan Lektemur Alpan ${ }^{2}$
}

${ }^{1}$ DDS, PhD. Department of Maxillofacial Surgery, Faculty of Dentistry, Erzincan Binali Yıldırım University, Erzincan, Turkey

${ }^{2}$ DDS, PhD. Department of Periodontology, Faculty of Dentistry, Pamukkale University, Denizli, Turkey

Correspondence:

Pamukkale University Faculty of Dentistry

Department of Periodontology Kinıklı Campus

20160 Denizli, Turkey

ysnlpn@gmail.com

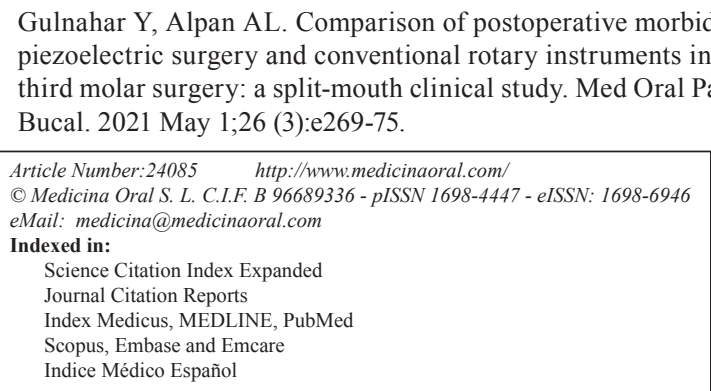

\begin{abstract}
Background: The extraction of impacted third molar teeth is a common procedure in maxillofacial surgery. The aim of this study was to compare of piezoelectric surgical technique with the one with conventional rotary instruments in terms of edema, trismus and pain, in mandibular third molar surgery.

Material and Methods: 20 individuals with symmetrically impacted lower mandibular third molars and 40 teeth were included in the study. Third molars on the left side of each patient were removed with piezosurgery, while the counterparts on the right side were removed with conventional rotary instruments. Postoperatively, the same antibiotic, analgesic, and mouthwash were recommended to both groups. Ultrasound, edema, trismus measurements were performed before surgery, postoperative, postoperative day 2 and postoperative day 7. VAS scale was used to evaluate the pain.

Results: The average age of 20 individuals included in the study was found to be $21.85 \pm 3.08$ years. The operation time of the individuals who underwent the surgery with conventional rotary instruments was found to be 12 minutes $31.70 \pm 167.03$ seconds, and the operation time in the Piezosurgery group was 19 minutes $10.60 \pm 306.59$ seconds. There was no significant difference between the two groups in terms of trismus, edema, and pain. Conclusions: Piezosurgery is a safe method that can be used in molar removal, but in this split-mouth study, it is not found advantageous in terms of postoperative morbidity due to the longer working time compared to the one performed with conventional rotary instruments.
\end{abstract}

Key words: Edema, impacted third molar, pain, piezosurgery, trismus. 


\section{Introduction}

Full or partially impacted teeth in the mouth cause various complications such as pericoronitis, caries, periodontal problems of adjacent molar, germination disorders and orthodontic problems (1). Therefore, third molar surgery is the most applied procedure in maxillofacial surgery. Main complications regarding third molar dental surgery are trismus, edema and pain. It is argued that the factors that combine these complications are complex, but the main factor is due to the inflammatory process caused by surgical trauma (2).

Various researchers have carried out various studies to reduce postoperative morbidity. These studies are related to; preoperative use of antibiotics, various flap techniques, using conventional rotary instruments with high or low speed, performing drain application to the operation site or not (3), postoperative use of ice (4), using systemic or topical cortisone $(5,6)$ and using $\mathrm{Er}$ -YAG lasers (7).

In maxillofacial surgery, bone incisions are made with mechanical tools such as saws and burs. Mechanical tools have harmful effects due to the high amount of heat generated during the bone incision. This temperature may eventually cause marginal osteonecrosis, as it ultimately inhibits bone regeneration (8). Therefore, in the past, serious experimental efforts have been made to develop better osteotomie devices in response to the need for more precise and safe osteotomie in bone surgery (9).

In the field of dentistry, ultrasonic devices found use mainly in periodontology and endodontics after the cutting effects of high-frequency sound waves on dental hard tissues were observed in 1953. Piezosurgery is a technique that enables safe and effective osteotomies using ultrasonic vibrations. Due to its micrometric and selective cutting, the piezosurgery unit provides a safe and sensitive osteotomy without causing osteonecrotic damage. The device works only on mineralized tissues while protecting soft tissue and maintaining blood supply $(9,10)$.

Minimally invasive surgery is essential for reducing tissue trauma and patient morbidity. In recent years, with the inclination of modern medicine towards minimally invasive surgery, the use of ultrasonic waves for bone insicions has gained importance in oral and maxillofacial surgery, on account of that ultrasonic micro-movements do not show any visible damage to adjacent soft tissues (11).

Working with ultrasound frequency, this device cuts hard tissue with micro-vibrations between 60 and 200 $\mathrm{mm} / \mathrm{s}$ and in the frequency range of 24 to $29 \mathrm{kHz}$; and it solely targets mineralized tissues without damaging nerves, vessels and soft tissues (12). The tissue-selective and less heat-generating structure of piezosurgery cause less bleeding. It is advantageous to use piezosur- gery in plastic surgery procedures such as genioplasty or rhinoplasty with high aesthetic expectations (13). However, its main disadvantages include high cost and long operating time (14). In addition, in a histopathological study, it was found that piezosurgery caused less edema but more tissue necrosis than the conventional method (15). Based on the known advantages of piezosurgery, we therefore hypothesize that pieosurgery would reduce postoperative morbidity in terms of pain, edema, and trismus in mandibular third molar surgery. In this study, we aimed to determine the effects of piezoelectric and conventional surgical techniques on postoperative morbidity.

\section{Material and Methods}

Permission was obtained from the Ethics Committee of Cumhuriyet University Faculty of Dentistry (No. 26 dated 18/11/2008) for the study protocol. Our study was planned as a prospective split-mouth study. All procedures were performed in conformity with the rules of the Declaration of Helsinki. This study was performed on patients who applied to Sivas Cumhuriyet University Faculty of Dentistry, Department of Oral and Maxillofacial Diseases for the removal of impacted mandibular third molars. Only those who were over 18 and had no systemic disease were included in the study. The patients had similar type and class of impaction on both sides of mandible and the uniform thickness of the bone that must be removed seen in the panoramic image according the Pell and Gregory classification (16). Patients who were smoking, pregnant; who had chronic diseases or severe periodontitis; and who were using analgesics or anti-inflammatory were excluded. All operations were carried out by the same physician under the same surgical and sterilization disciplines, under equal operating conditions. A written informed consent was obtained from all of the patients.

Based on the reference article, a minimum of 18 people were included in each group, with 1.26 effect size and $95 \%$ power (17). In the study, 20 patients underwent an operation for the removal of 40 impacted mandibular third molars which were symmetrically impacted in both right and left jaws. The symmetries of the teeth were evaluated according to the radiographic views of the anatomical positions of the third molar (18). During the operations, impacted mandibular third molars on one side were extracted using conventional surgical methods while the rest on the other side were extracted with a piezosurgery unit (Esacrom, Bologna; Italy). Ascertaining random instrument sequence, coin toss method used for randomization. The period from taking the first patient to the recovery of the last patient is 6 months. At least four weeks of recovery period was expected in the same patient for the removal of the second tooth. 
- Surgery Procedure

The patients were asked to rinse the oral cavity with a $2 \%$ povidone-iodine solution 5 minutes before the procedure. During the operations, a local anesthetic agent containing $40 \mathrm{mg} / \mathrm{ml}$ Articaine $\mathrm{HCl}$ and $0.012 \mathrm{mg} /$ $\mathrm{ml}$ epinephrine $\mathrm{HCl}$ (Ultraca DS forte-Aventis Pharmaceutical Tic. Inc., Istanbul, Turkey) was used as a local anesthetic solution in all groups. After the anesthesia of the $\mathrm{n}$. alveolaris inferior and buccal nerve was achieved, the buccal flap was lifted with the "L" incision, the bone was removed with the help of a steel rod and fissure bur and the molar was extracted in conventional surgery group. In the piezosurgery group, piezosurgery (EMS, Piezon Master Surgery, Switzerland) unit installed with ES001 and ES005 inserts and the molar was extracted. The flap was closed primarily with a 3-0 silk suture. The time between the first incision and the last suture was recorded as the operation time. For infection control, when necessary, considering patients with penicillin allergy and stomach problems, and in order to ensure the standardization, clindamycin $\mathrm{HCl} 150 \mathrm{mg}$ (from Kline, Science, Istanbul, Turkey) 4x1, paracetamol $500 \mathrm{mg}$ (minoset, Bayer, Turkey) 3x1 and chlorhexidine gluconate \% 0.2 (Klorhex, Drogs, Ankara, Turkey) 2x1 were prescribed. The physical therapy method (cold therapy) was not recommended to any patient included in the study.

- Postoperative Edema

Postoperative edema was measured $(\mathrm{cm})$ the linear distances from the angle of the mandible to the lateral canthus of the eye and from the tragus to the corner of the mouth and from the tragus to the pogonion (19). Postoperative edema was also evaluated with ultrasonography which is noninvasive, painless, rapid and inexpensive technique without any known deleterious biological effects $(20,21)$. In order to evaluate the edema with ultrasound device, preoperative and postoperative soft tissue measurements were recorded at Sivas Cumhuriyet University Faculty of Medicine, Department of Radiology with the same device, by the same physician and with minimal pressure on the skin. For this, GE Logiq 9 (USA) brand, ultrasonography, and Sony (Japan) brand printer (UP-D897) devices were used. In each patient, the distance between the skin surface and the bone surface was measured from the fixed point marked on the skin in the sitting position and the patient's jaw muscles were not contracted, using the $10 \mathrm{MHz}$ probe before and after the operation on the 2nd and 7th day. While determining the fixed point, the probe was placed $15 \mathrm{~mm}$ in front of the angulus mandible, which is parallel to the lower edge of the mandible and placed $15 \mathrm{~mm}$ in front of the angulus mandible, was taken as reference. In other words, the $30-35 \mathrm{~mm}$ front side of the angulus mandible was determined as the reference point. To increase the contact between the probe and the skin, the gel was ap- plied to the masseter muscle area to be measured.

- Evaluation of Trismus

Caliper (VIS-Poland) was used to measure the trismus degree. The evaluation of the postoperative trismus, as it was done to check edema, was performed preoperatively and also on the 2 nd and 7 th postoperative days by the method of measuring mouth opening. The patients were asked to open their mouths as much as possible, and the distance between the incisal edges of the upper and lower central incisors was measured in millimeters with the help of a caliper. This process was repeated 3 times for each measurement and the arithmetic mean of the values found was taken.

- Postoperative Pain

A visual analog scale (VAS) of $100 \mathrm{~mm}$ was used to evaluate postoperative pain. 0 means "no pain" in this scale while 100 equals the worst pain imaginable (22). The patients were asked to mark the amount of pain at this scale on the 30th minute, 3rd, 6th, 9th and 24th hours following the operation and also on the 2nd, 3 rd,4th,5th,6th and 7th days. At the end of the operation, patients were given forms that gave information about postoperative care. In another form, the patients were asked to rate the severity of pain at the specified hours.

- Statistical analysis

SPSS v.21.0 (SPSS Inc., Chicago, IL) was used to analyze the data. Data normality was tested using the Shapiro-Wilk test. As VAS measurements are nonparametric, related samples Wilcoxon test was used for measurements of the same group. Repeated measurements ANOVA used for trismus and edema measurements. For paired comparisons, the Mann-Whitney U test for nonparametric measurements and independent sample $t$ test for parametric measurements were used. Statistical significance was established at $\mathrm{p}<0.05$.

\section{Results}

20 individuals with 40 symmetrically impacted mandibular third molars were included in the study. All patients were observed during the study. The average age of 20 individuals included in the study was $21.85 \pm 3.08$ years. $7(35 \%)$ of these individuals were male and 13 $(65 \%)$ were female. The conventional method was used for $8(40 \%)$ right and $12(60 \%)$ left mandibular third molars whereas the piezosurgery method was used for 12 $(60 \%)$ right and $8(40 \%)$ left mandibular third molars.

- Operation Durations

The average operation time was 12 minutes in the conventional group and the average operation time was 19 minutes in the piezosurgery group. There was a significant difference between these two methods in terms of operation time $(\mathrm{p}=0.001, \mathrm{p}<0.05)$.

- Evaluation of Trismus

In the postoperative 1st day measurement, $6.38 \%$ and 
$6.46 \%$ reduction in mouth opening were observed in the conventional and piezosurgery groups, respectively. In the postoperative 2 nd day measurements, there was a $34.18 \%$ and $26.92 \%$ reduction in mouth opening compared to the initial measurements. On the 7 th postoperative day, this reduction was reduced to $22.1 \%$ and $13.1 \%$. When all time periods are considered, the difference between the two groups was not statistically significant ( $\mathrm{p}>0.05)$ (Fig. 1)

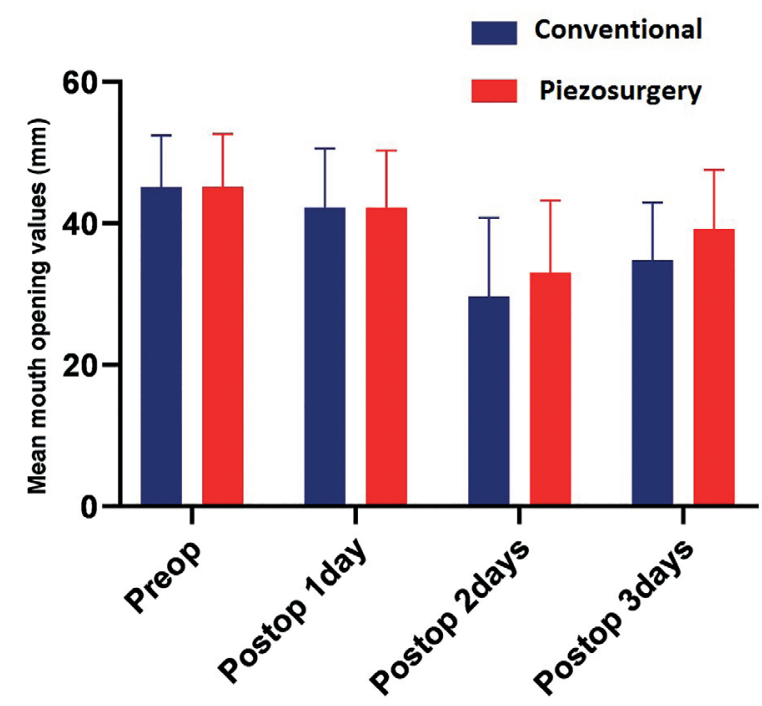

Fig. 1: Quae. Ro voluption perunt utendia vel ipsum que parum.

- Evaluation of Edema

According to the average of the measurements of TragusMouth Corner, Tragus-Jaw Tip, Angulus-Eye Corner, which we made for the measurement of edema, on preoperative, postoperative, postoperative 2 nd day and 7 th day; an increase of $0.25 \%$ in the postoperative measurement, $2.18 \%$ in the postoperative 2 nd day measurement, and $0.01 \%$ in the postoperative 7 th day measurement were observed in the conventional group. In the piezo group, an increase of $0.08 \%$ was observed in the postoperative measurement, $0.93 \%$ in the postoperative 2 nd day measurement and $0.16 \%$ in the postoperative 7 th day (Table 1 ).
- Evaluation of Edema with Ultrasound

In order to evaluate the edema, ultrasound measurements of the masseter muscle and subcutaneous were performed on preoperative, postoperative and postoperative 2nd day. When compared to the preoperative measurements $(6.15 \mathrm{~mm})$ by taking the average of the two values; In group A, an increase of $16.5 \%$ was observed in the postoperative measurement $(7.17 \mathrm{~mm})$ and a $46.8 \%$ increase in the postoperative 2 nd day measurement (9.03). In group B, an increase of $11.5 \%$ was observed in the postoperative measurement $(7.45 \mathrm{~mm})$ compared to the preoperative measurement $(6.68 \mathrm{~mm})$, and an increase of $26.8 \%$ in the postoperative second day measurement $(8.47 \mathrm{~mm})$ (Fig. 2).

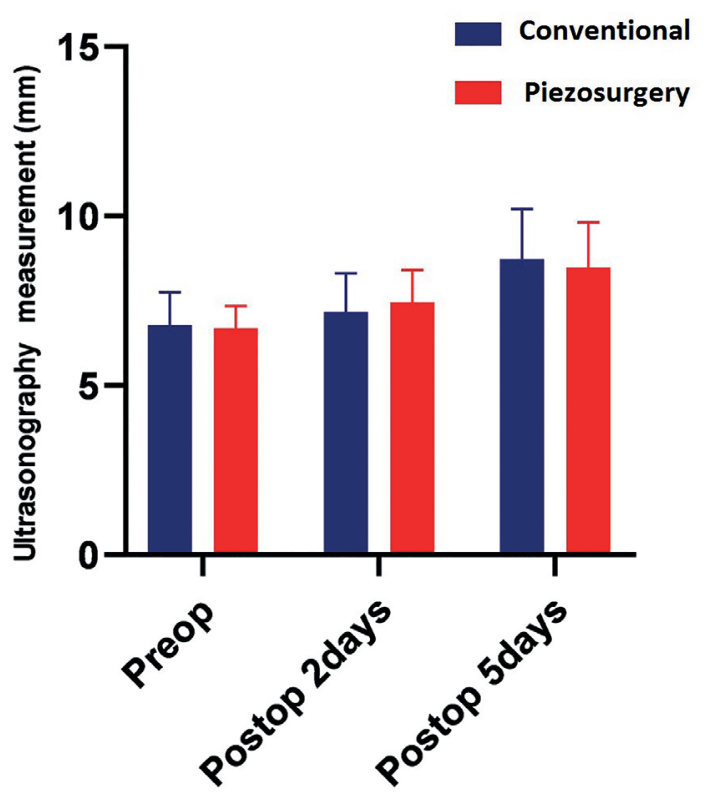

Fig. 2: Ultrasonography data of groups.

- Evaluation of Pain

Considering the findings related to pain, there was a significant difference between the two groups at 3rd hour and 9 th hour $(\mathrm{p}<0.05)$. In the data up to 24 hours, the conventional method created more pain than piezo-

Table 1: Result of edema measurements.

\begin{tabular}{|c|c|c|c|c|c|c|}
\hline & & $\begin{array}{l}\text { Preoperative } \\
(\mathrm{mm})\end{array}$ & $\begin{array}{c}1^{\text {st }} \text { day } \\
(\mathrm{mm})\end{array}$ & $\begin{array}{c}2^{\text {nd }} \text { day } \\
(\mathrm{mm})\end{array}$ & $\begin{array}{c}7^{\text {th }} \text { day } \\
(\mathrm{mm})\end{array}$ & Pvalue \\
\hline \multirow{2}{*}{$\begin{array}{l}\text { Tragus-Mouth } \\
\text { Corner }\end{array}$} & Conventional & $10.82 \pm 0.51$ & $10.84 \pm 0.69$ & $11.09 \pm 0.53$ & $10.83 \pm 0.52$ & \multirow[t]{2}{*}{$>0.05$} \\
\hline & Piezosurgery & $10.76 \pm 0.57$ & $10.78 \pm 0.58$ & $10.88 \pm 0.58$ & $10.79 \pm 0.79$ & \\
\hline \multirow{2}{*}{$\begin{array}{l}\text { Tragus-Jaw } \\
\text { Tip }\end{array}$} & Conventional & $14.55 \pm 0.52$ & $14.59 \pm 0.52$ & $14.83 \pm 0.54$ & $14.54 \pm 0.54$ & \multirow[t]{2}{*}{$>0.05$} \\
\hline & Piezosurgery & $14.51 \pm 0.67$ & $14.52 \pm 0.67$ & $14.62 \pm 0.68$ & $14.53 \pm 0.67$ & \\
\hline \multirow{2}{*}{$\begin{array}{l}\text { Angulus-Eye } \\
\text { Corner }\end{array}$} & Conventional & $10.31 \pm 0.72$ & $10.34 \pm 0.73$ & $10.54 \pm 0.54$ & $10.33 \pm 0.71$ & \multirow[t]{2}{*}{$>0.05$} \\
\hline & Piezosurgery & $10.16 \pm 0.52$ & $10.16 \pm 0.52$ & $10.25 \pm 0.57$ & $10.17 \pm 0.53$ & \\
\hline
\end{tabular}

Values are presented as means \pm SD. The relationships between groups and results were assessed by one-way ANOVA. 


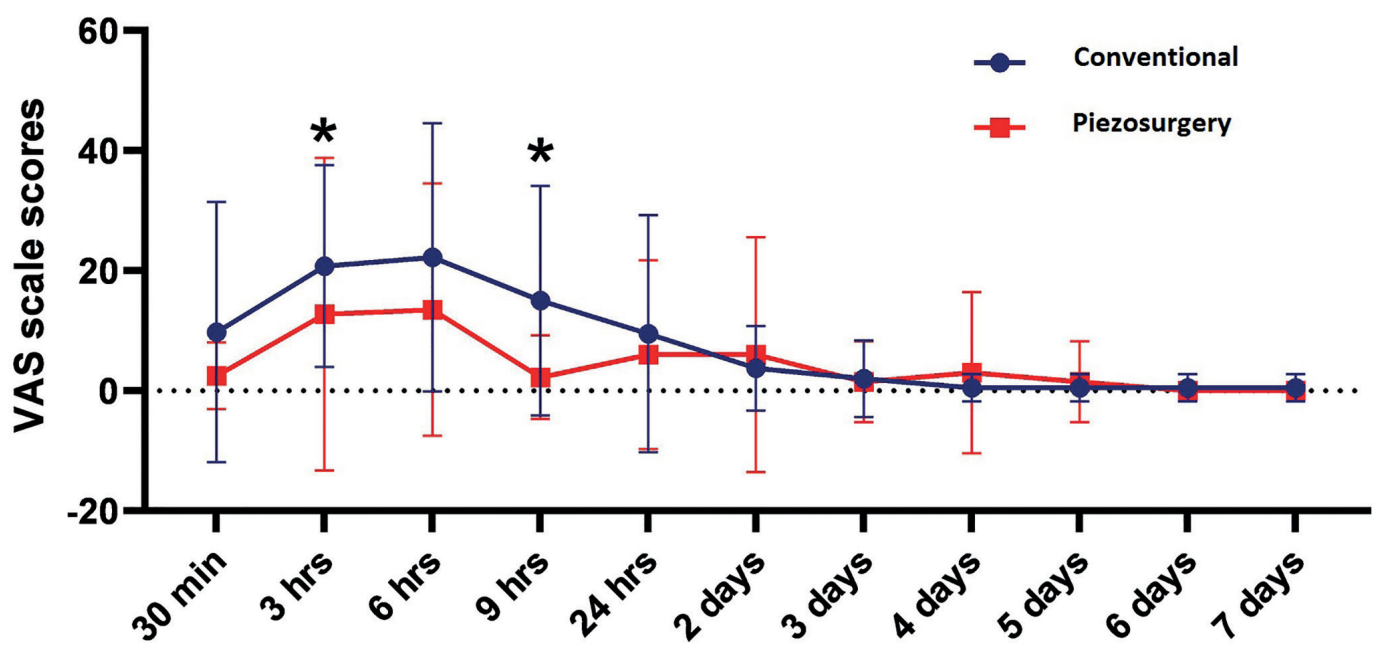

Fig. 3: Mean VAS values of groups. ${ }^{*} \mathrm{p}<0.05$ between two groups.

surgery, but this curve continued at almost the same level after 24 hours. The pain level reached a peak in 6 hours in both groups and continued to decrease after that (Fig. 3). In the data of the first 24 hours, the conventional method created more pain than the piezosurgery method, but this curve continued at almost the same after 24 hours. The pain reached its peak level in the first 6 hours in both groups and continued to decrease after that (Fig. 3).

\section{Discussion}

In maxillofacial surgery, the extraction of mandibular third molars is often performed. While conventional rotary handpieces are used for these surgeries, the use of piezosurgery has gained popularity with its selective cutting feature. In this split-mouth study, two surgical methods were compared with their advantages and disadvantages in terms of complications after mandibular third molars extraction, and according to the results of the study, no significant difference was found between the two methods in terms of postoperative edema and trismus. When the postoperative pain was evaluated, less pain was observed in the piezosurgery group at the 3rd and 9th hours between the two groups.

Piezosurgery has many advantages mentioned in the literature. First of all, it does not produce excessive heat during the operation; secondly, continuous irrigation makes the operation area more visible, thirdly, it does not damage the soft tissues, especially inferior alveolar nerve, periosteum, Schneiderian membrane, and oral mucosa, which are close to the surgery area, and it reduces bleeding in the surgical area. Many studies are investigating postoperative morbidity after the removal of mandibular third molars. Pain, swelling, and trismus were generally examined in these studies. In a study by
Goyal et al. (23), it was reported that the use of piezosurgery significantly reduced postoperative pain, swelling, and trismus. In a similar study, it was revealed that piezosurgery caused an increase in the quality of life of patients and decreased postoperative pain, trismus, and swelling (24). In a split-mouth study, Menziletoglu et al. (25) investigated the effect of piezosurgery on postoperative pain, swelling, trismus and the comfort of the patient, and according to the results, piezosurgery did not provide a superiority over conventional method.41 In our study, only 3 hrs and 9 hrs VAS results was significantly lower in the favor of piezosurgery but all other investigated data in terms of postoperative pain, trismus and edema, differences were insignificant. While other evaluations, other than VAS, are subjective, VAS is a personal and objective evaluation method. Therefore, different results can be found in studies involving VAS. Similar to our study results, Taskın et al. (26) did not find a statistically significant difference between the two groups in terms of postoperative edema in a cohort of 90 patients (45 conventional and 45 piezosurgery). Bhati et al. (27) compared piezosurgery and conventional methods in 30 patients; parameters such as mouth opening (interincisal opening), pain (visual analog scale VAS score), swelling, the incidence of dry socket, paresthesia and duration of surgery were examined and piezosurgery showed a significant difference only in terms of VAS. The prolongation of the operation time compared to the conventional method is among the most important disadvantages of piezosurgery (14). In our study, the piezosurgery time was found to be significantly higher than the conventional method. Longer exposure of the bone may limit the advantages of this technique. Additionally, performing osteotomy on mandibular third molars and entering that narrow area may 
take longer and extend the duration of the operation due to the design of the piezosurgery inserts. Longer surgery causes more manipulation of the tissues and, consequently, results more inflammatory responses (28). The duration of surgery is predictive not only for the amount of edema but also for pain and trismus intensity. This is due to intraoperative complications directly related to a larger trauma or an increase in the duration of surgery (28).

Some authors claim that facial surgery in young individuals results in less difficulty in the procedure and, consequently, less surgical trauma and less edema. Elderly patients have a prolonged inflammatory process, and therefore there is a slower reduction in edema (29). In our study, the mean age of the patients was $21.85 \pm$ 3.08 years and they were quite young, and the absence of a significant difference in edema and trismus may be due to these individuals' immune systems being better than the elderly and faster resolution of the inflammatory process. Surely postoperative edema and complications are not linked to only one point. The surgeon's ability to use that instrument efficiently also gains importance in this regard. Since piezosurgery units are more expensive than conventional rotary instruments, not every surgeon may have used this unit sufficiently. Furthermore, since all training are given primarily with conventional rotary instruments, the surgeon's experience may be more in that regard and may cause a decrease in postoperative complications even though there is an indirect effect (30).

Piezosurgery is a safe alternative method that can be used for the removal of impacted mandibular third molars. Despite this fact, postoperative pain did not provide a significant benefit in terms of edema and trismus. The prolonged surgical process, the price of these units and the need for intensive surgical experience are the disadvantages of piezosurgery. However, thanks to its selective cutting feature, piezosurgery can be preferred if there is a third molar near the anatomical formations.

\section{References}

1. Liu J, Hua C, Pan J, Han B, Tang X. Piezosurgery vs conventional rotary instrument in the third molar surgery: A systematic review and meta-analysis of randomized controlled trials. J Dent Sci. 2018;13:342-9.

2. Laureano Filho JR, de Oliveira e Silva ED, Batista CI, Gouveia FM. The influence of cryotherapy on reduction of swelling, pain and trismus after third-molar extraction: a preliminary study. J Am Dent Assoc. 2005;136:774-8.

3. Cerqueira PR, Vasconcelos BC, Bessa-Nogueira RV. Comparative study of the effect of a tube drain in impacted lower third molar surgery. J Oral Maxillofac Surg. 2004;62:57-61.

4. van der Westhuijzen AJ, Becker PJ, Morkel J, Roelse JA. A randomized observer blind comparison of bilateral facial ice pack therapy with no ice therapy following third molar surgery. Int J Oral Maxillofac Surg. 2005;34:281-6.

5. Alexander RE, Throndson RR. A review of perioperative corticosteroid use in dentoalveolar surgery. Oral Surg Oral Med Oral Pathol Oral Radiol Endod. 2000;90:406-15.
6. Graziani F, D'Aiuto F, Arduino PG, Tonelli M, Gabriele M. Perioperative dexamethasone reduces post-surgical sequelae of wisdom tooth removal. A split-mouth randomized double-masked clinical trial. Int J Oral Maxillofac Surg. 2006;35:241-6.

7. Abu-Serriah M, Critchlow H, Whitters CJ, Ayoub A. Removal of partially erupted third molars using an Erbium (Er):YAG laser: a randomised controlled clinical trial. Br J Oral Maxillofac Surg. 2004;42:203-8.

8. Kerawala CJ, Martin IC, Allan W, Williams ED. The effects of operator technique and bur design on temperature during osseous preparation for osteosynthesis self-tapping screws. Oral Surg Oral Med Oral Pathol Oral Radiol Endod. 1999;88:145-50.

9. Schaller BJ, Gruber R, Merten HA, Kruschat T, Schliephake H, Buchfelder M, et al. Piezoelectric bone surgery: a revolutionary technique for minimally invasive surgery in cranial base and spinal surgery?. Technical note. Neurosurgery. 200;57:E410.

10. Gruber RM, Kramer FJ, Merten HA, Schliephake H. Ultrasonic surgery--an alternative way in orthognathic surgery of the mandible. A pilot study. Int J Oral Maxillofac Surg. 2005;34:590-3.

11. Robiony M, Polini F, Costa F, Zerman N, Politi M. Ultrasonic bone cutting for surgically assisted rapid maxillary expansion (SARME) under local anaesthesia. Int J Oral Maxillofac Surg. 2007;36:267-9.

12. Kotrikova B, Wirtz R, Krempien R, Blank J, Eggers G, Samiotis A, et al. Piezosurgery--a new safe technique in cranial osteoplasty? Int J Oral Maxillofac Surg. 2006;35:461-5.

13. Bertossi D, Albanese M, Mortellaro C, Malchiodi L, Kumar N, Nocini R, et al. Osteotomy in Genioplasty by Piezosurgery. J Craniofac Surg. 2018;29:2156-9.

14. Meller C, Havas TE. Piezoelectric technology in otolaryngology, and head and neck surgery: a review. J Laryngol Otol. 2017;131:S12-S8. 15. Demirbilek N, Evren C. Is Piezoelectric Surgery Really Harmless to Soft Tissue? J Craniofac Surg. 2019;30:1966-9.

16. Lima CJ, Silva LC, Melo MR, Santos JA, Santos TS. Evaluation of the agreement by examiners according to classifications of third molars. Med Oral Patol Oral Cir Bucal. 2012;17:e281-6.

17. Piersanti L, Dilorenzo M, Monaco G, Marchetti C. Piezosurgery or conventional rotatory instruments for inferior third molar extractions? J Oral Maxillofac Surg. 2014;72:1647-52.

18. Rullo R, Addabbo F, Papaccio G, D'Aquino R, Festa VM. Piezoelectric device vs. conventional rotative instruments in impacted third molar surgery: relationships between surgical difficulty and postoperative pain with histological evaluations. J Craniomaxillofac Surg. 2013;41:e33-8

19. Patil C, Jadhav A, K R, Bhola N, Borle RM, Mishra A. "Piezosurgery vs bur in impacted mandibular third molar surgery: Evaluation of postoperative sequelae". J Oral Biol Craniofac Res. 2019;9:259-62. 20. Rama Mohan K, Koteswara Rao N, Leela Krishna G, Santosh Kumar V, Ranganath N, Vijaya Lakshmi U. Role of ultrasonography in oral and maxillofacial surgery: a review of literature. J Maxillofac Oral Surg. 2015;14:162-70.

21. Oeppen RS, Gibson D, Brennan PA. An update on the use of ultrasound imaging in oral and maxillofacial surgery. Br J Oral Maxillofac Surg. 2010;48:412-8.

22. Lektemur Alpan A, Torumtay Cin G. PRF improves wound healing and postoperative discomfort after harvesting subepithelial connective tissue graft from palate: a randomized controlled trial. Clin Oral Investig. 2020;24:425-36.

23. Goyal M, Marya K, Jhamb A, Chawla S, Sonoo PR, Singh V, et al. Comparative evaluation of surgical outcome after removal of impacted mandibular third molars using a Piezotome or a conventional handpiece: a prospective study. Br J Oral Maxillofac Surg. 2012;50:556-61.

24. Arakji H, Shokry M, Aboelsaad N. Comparison of Piezosurgery and Conventional Rotary Instruments for Removal of Impacted Mandibular Third Molars: A Randomized Controlled Clinical and Radiographic Trial. Int J Dent. 2016;2016:8169356.

25. Menziletoglu D, Basturk F, Isik BK, Esen A. A prospective splitmouth clinical study: comparison of piezosurgery and conventional rotary instruments in impacted third molar surgery. Oral Maxillofac 
Surg. 2020;24:51-5.

26. Taskin U, Batmaz T, Erdil M, Aydin S, Yucebas K. The comparison of edema and ecchymosis after piezoelectric and conventional osteotomy in rhinoplasty. Eur Arch Otorhinolaryngol. 2017;274:861-5. 27. Bhati B, Kukreja P, Kumar S, Rathi VC, Singh K, Bansal S. Piezosurgery versus Rotatory Osteotomy in Mandibular Impacted Third Molar Extraction. Ann Maxillofac Surg. 2017;7:5-10.

28. de Santana-Santos T, de Souza-Santos a A, Martins-Filho PR, da Silva LC, de Oliveira ESED, Gomes AC. Prediction of postoperative facial swelling, pain and trismus following third molar surgery based on preoperative variables. Med Oral Patol Oral Cir Bucal. 2013;18:e65-70.

29. Perez-Gonzalez JM, Esparza-Villalpando V, Martinez-Rider R, Noyola-Frias MA, Pozos-Guillen A. Clinical and Radiographic Characteristics as Predictive Factors of Swelling and Trismus after Mandibular Third Molar Surgery: A Longitudinal Approach. Pain Res Manag. 2018;2018:7938492.

30. Jerjes W, El-Maaytah M, Swinson B, Banu B, Upile T, D'Sa S, et al. Experience versus complication rate in third molar surgery. Head Face Med. 2006;2:14.

\section{Funding}

No grants were received for this study that was supported solely by our institution.

\section{Conflict of interest}

The authors declare that they have no conflict of interests.

\section{Ethics}

Permission was obtained from the Ethics Committee of Cumhuriyet University Faculty of Dentistry (No. 26 dated 18/11/2008) for the study protocol.

\section{Authors contributions}

YG planned and performed all experimental procedures of the study. ALA performed the statistical analyzes, preparation and writing of the manuscript. 